\title{
Can Temporomandibular Joint Disorders Be Diagnosed Beforehand by Assessment of Postural Irregularities?
}

\author{
(1) Canan Dağ'1, (1) Akif Demirel2, (1) Nurhan Özalp² \\ PPrivate Pediatric Dentist, Ankara, Turkey \\ ${ }^{2}$ Ankara University Faculty of Dentistry, Department of Pediatric Dentistry, Ankara, Turkey
}

\begin{abstract}
Aim: In many studies, the relationship between postural status and temporomandibular disorders (TMD) have been investigated, however there is no consensus on this regard. The aim of this study was to investigate the relationship between postural irregularities forward head posture (FHP) and different shoulder levels (DSL) and TMD prevalence in different dentition stages.

Materials and Methods: This study, which included children between 4 and 14 years of age attending public schools in Ankara, was planned as a cross-sectional study. Temporomandibular joint (TMJ) was examined intra- and extra-orally. After the examination, the relationship between TMD and body posture (FHP and DSL) was investigated. For statistical analysis, chi-square test and Fisher's exact test were used with significance level of $p<0.05$.

Results: FHP and DSL were statistically related to TMD $(p<0.05)$. In primary dentition, there was no significant relation between FHP and $T M D$, however, DSL were found to be significantly related to TMD $(p<0.05)$. In mixed dentition, both of these parameters were statistically related to TMD $(p<0.05)$. However, in the permanent dentition, there was no relationship between body posture and TMD.

Conclusion: Since there is a strong correlation between postural irregularities and TMD especially in the mixed dentition stage, TMJ and postural status of pediatric patients should be examined as early as possible in the stages of the life. In this regard, the awareness of pediatricians and pediatric dentists to this matter needs to be improved.
\end{abstract}

Keywords: TMJ, TMD, head posture, body posture

\section{Introduction}

The temporomandibular joint (TMJ) is an ellipsoid variety of the synovial joints forming a bicondylar articulation. TM) includes a disk, fibrous capsule, synovial membrane and ligaments (1). Temporomandibuler disorders (TMD) are defined as neuromuscular and musculoskeletal problems characterized by TMJ, masticatory muscles and clinical findings associated with the related structures (2). TMD are known as functional irregularities of the general masticatory system, TMD associated disk displacements and degenerative and inflammatory diseases of these structures $(3,4)$.

TMD occurs with multiple aetiological factors (5) and common causes are parafunctional habits such as macrotrauma, bruxism and clenching, skeletal and occlusal disorders, psychosocial factors and systemic factors (6). The prevalence of TMD is highly variable due to differences in populations studied in children and adolescents (6). In general, clinical symptoms associated 
with TMD are less common in the primary dentition period than in the mixed and permanent dentition periods (7).

It is known that TMD is positively related to head and body posture (8-13). The body posture is a position associated with muscle activation regulated by the central nervous system (14). The biomechanical organization of the body posture is regulated by the functional integration of the various body structures, and the changes in any biological subunit result in the differentiation and reorganization of the postural control systems $(11,13)$.

The stomatognathic system includes structures which have an important role in postural control such as the lower and upper jaw, dental arches, neurovascular soft tissues and muscle groups related to TMJ (14). The muscular groups of the stomatognathic system belong to the muscle groups of the neck and all units of the muscular chain are related to each other. As a matter of fact, TMJ is a structure which is connected to the neck region via the muscular and ligamental structures and this functional unit is called "cranio-cervico-mandibular" system $(14,15)$. In light of these points, any disorder of the muscular component leads to a reorganization of other subunits (11). Therefore, the relationship between body posture and the incidence of TMD needs to be clearly defined $(11,16)$ in order to provide the optimal treatment for TMD patients.

The aim of this study is to investigate the relation between postural irregularities and TMD prevalence in different dentition stages. The secondary goal of this research is to improve the awareness of pediatric dentists and pediatricians in order to facilitate the early diagnosis of TMD by additional examinations which include postural status.

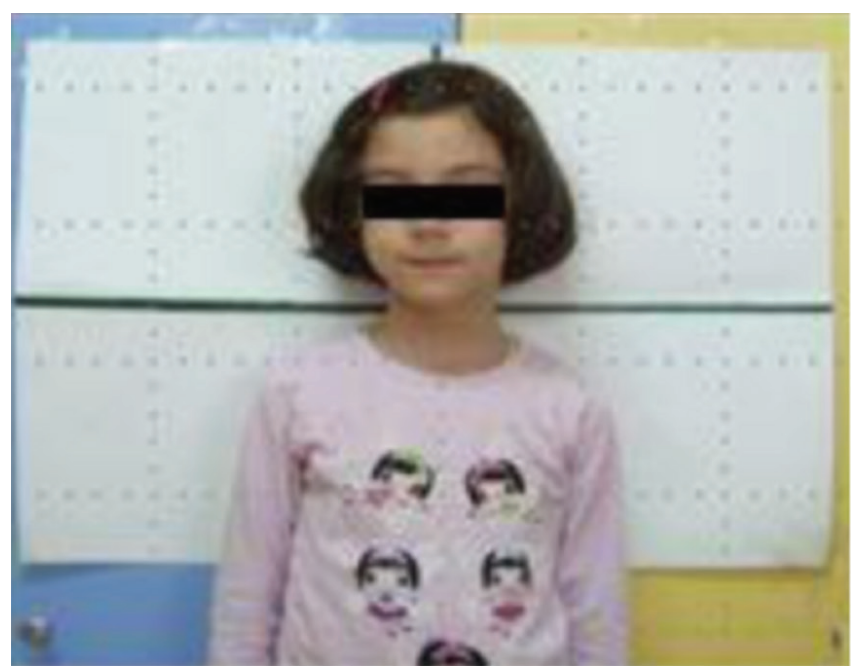

Figure 1. Photos taken for posture evaluation and guide lines

\section{Materials and Methods}

\section{Sample Selection and Ethical Approval}

This study, which included children between 4-14 years of age attending public schools in Ankara, was planned as a cross-sectional study including intra-oral and extra-oral examinations. The study protocol was approved by the Ethics Committee of Ankara University (approval number: 150/2). The participants of the study were selected based on a voluntary basis and the parents of the children who participated in the study gave informed written and verbal consent. After detailed information was provided, informed consent forms were signed by the parents. A power analysis was performed to determine the sample size by using the number of children at different dentition groups included in the study (Table I).

\section{Evaluation of the Postural Status}

A total of 6 photographs (3 profile, 3 facade) were taken for the postural evaluation of the children. After the participants were positioned on a flat surface, photographs were taken from a distance of 2 meters. Right and left shoulder location levels were evaluated in the facade photographs and the average value of the photographs were recorded as a result. Similarly, in the photographs taken in profile, the location of the shoulder and the ear was evaluated and averages of photographs were

\begin{tabular}{|l|l|}
\hline \multicolumn{2}{|l|}{ Table I. Sample sizes of different dentition groups } \\
\hline Ages & $\mathbf{n}$ \\
\hline 4-6 (Primary dentition) & 298 \\
\hline 7-11 (Mixed dentition) & 669 \\
\hline 12-14 (Permanent dentition) & 408 \\
\hline Total & 1375 \\
\hline
\end{tabular}

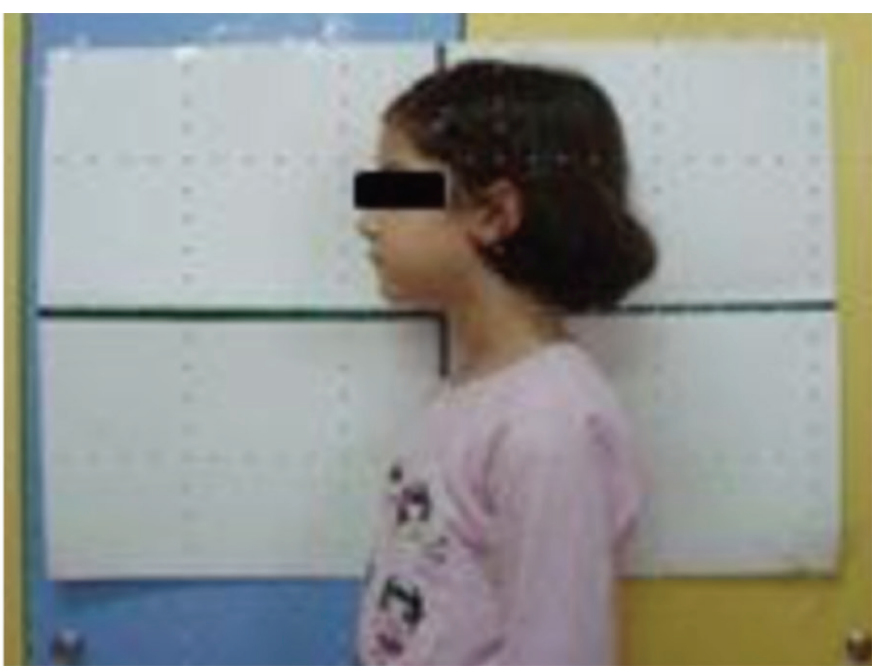


recorded. Digital guide lines were used in the evaluation of photographs (Figure 1). The shoulder levels were examined based on a horizontal line and in case of inequality in shoulder location, it was recorded as different shoulder level (DSL). In the photographs taken from the profile, the guide line passing over the shoulders was taken as the criterion and the anterior head position were recorded as forward head posture (FHP).

\section{Examination of Temporomandibular Joint and Diagnose of Temporomandibular Disorders}

The examination of TMJ and diagnosis of TMD were performed by observing the bilateral palpation of the skin, muscles and joints and all movements of the mandible.

Table II. Presence of postural irregularities in different dentition stages

\begin{tabular}{|l|l|l|}
\hline & FHP (\%) & DSL (\%) \\
\hline Primary dentition & 5.4 & 5.4 \\
\hline Mixed dentition & 5.8 & 5.5 \\
\hline Permanent dentition & 6.4 & 6.9 \\
\hline
\end{tabular}

FHP: Forward head posture, DSL: Different shoulder levels
Palpation of the joint was carried out intra-orally and extra-orally. Thus, the presence of tenderness on palpation and irregularities of lower jaw movements were recorded as "tenderness of masticatory muscles and TMJ". The presence of any findings such as deflection, deviation, disk displacement and joint sounds (clicking, popping, and crepitation) in TMJ examination were recorded as TMD. After this detailed examination, mouth opening capacity, maximum mouth opening, laterotrusion, retrusion, protrusion and presence of pain findings were recorded.

\section{Statistical Analysis}

The relationship between postural status and TMD in different dentition stages were analyzed using chi-square test and Fisher's exact test with a significance level of $p<0.05$.

\section{Results}

The percentage distribution of the postural irregularities based on FHP and DSL in different dentition stages is shown in Table II.

The relationship between postural status and TMD presence in primary, mixed and permanent dentition is given

\begin{tabular}{|c|c|c|c|c|c|c|c|c|c|c|}
\hline & & & \multicolumn{6}{|c|}{ TMD } & \multirow{3}{*}{ Chi-square test } & \multirow{3}{*}{$p$ value } \\
\hline & & & \multicolumn{2}{|c|}{ Absence } & \multicolumn{2}{|c|}{ Presence } & \multicolumn{2}{|c|}{ Total } & & \\
\hline & & & $\mathbf{n}$ & $\%$ & $\mathbf{n}$ & $\%$ & $\mathbf{n}$ & $\%$ & & \\
\hline \multirow{6}{*}{ Primary dentition } & \multirow{3}{*}{ FHP } & Absence & 267 & 94.68 & 15 & 5.32 & 282 & 100 & \multirow{3}{*}{ Fisher's exact test } & \multirow{3}{*}{0.063} \\
\hline & & Presence & 13 & 81.25 & 3 & 18.75 & 16 & 100 & & \\
\hline & & Total & 280 & 93.96 & 18 & 6.04 & 298 & 100 & & \\
\hline & \multirow{3}{*}{ DSL } & Absence & 268 & 95.04 & 14 & 4.96 & 282 & 100 & \multirow{3}{*}{ Fisher's exact test } & \multirow{3}{*}{$0.011^{*}$} \\
\hline & & Presence & 12 & 75.00 & 4 & 25.00 & 16 & 100 & & \\
\hline & & Total & 280 & 93.96 & 18 & 6.04 & 298 & 100 & & \\
\hline \multirow{6}{*}{ Mixed dentition } & \multirow{3}{*}{ FHP } & Absence & 553 & 87.78 & 77 & 12.22 & 630 & 100 & \multirow{3}{*}{27.381} & \multirow{3}{*}{$0.000^{*}$} \\
\hline & & Presence & 22 & 56.41 & 17 & 43.59 & 39 & 100 & & \\
\hline & & Total & 575 & 85.95 & 94 & 14.05 & 669 & 100 & & \\
\hline & \multirow{3}{*}{ DSL } & Absence & 552 & 87.34 & 80 & 12.66 & 632 & 100 & \multirow{3}{*}{16.325} & \multirow{3}{*}{$0.000^{*}$} \\
\hline & & Presence & 23 & 62.16 & 14 & 37.84 & 37 & 100 & & \\
\hline & & Total & 575 & 85.95 & 94 & 14.05 & 669 & 100 & & \\
\hline \multirow{6}{*}{ Permanent dentition } & \multirow{3}{*}{ FHP } & Absence & 317 & 82.98 & 65 & 17.02 & 382 & 100 & \multirow{3}{*}{ Fisher's exact test } & \multirow{3}{*}{0.107} \\
\hline & & Presence & 18 & 69.23 & 8 & 30.77 & 26 & 100 & & \\
\hline & & Total & 335 & 82.11 & 73 & 17.89 & 408 & 100 & & \\
\hline & \multirow{3}{*}{ DSL } & Absence & 316 & 83.16 & 64 & 16.84 & 380 & 100 & \multirow{3}{*}{3.180} & \multirow{3}{*}{0.075} \\
\hline & & Presence & 19 & 67.86 & 9 & 32.14 & 28 & 100 & & \\
\hline & & Total & 335 & 82.11 & 73 & 17.89 & 408 & 100 & & \\
\hline
\end{tabular}

TMD: Temporomandibular disorders, FHP: Forward head posture, DSL: Different shoulder levels, *Statistically significant difference 
in Table III. According to the results, there was no relation between TMD and the FHP in primary dentition, while the presence of TMD was found to be statistically significant in DSL $(p<0.05)$. Additionally, the presence of TMD was found to be statistically significant $(p<0.05)$ in both postural irregularities in mixed dentition. In permanent dentition, both postural irregularities were not statistically related to TMD. Nevertheless, it has been determined that the rate of TMD was higher in the individuals in whom the postural irregularities were observed.

The rate of presence of TMD was $34.6 \%$ in the presence of FHP, whereas the rate of TMD in normal head position was $12.1 \%$. The presence of TMD was statistically significantly higher in the presence of FHP $(p<0.05)$ (Table IV). Additionally, the incidence of TMD was $33.3 \%$ and $12.2 \%$ in patients with and without DSL, respectively. The presence of TMD was statistically significantly higher in the presence of DSL ( $p<0.05)$ (Table IV).

\section{Discussion}

TMD are common diseases in children and adolescents being at least as prevalent as for adults (17-20). TMD develop with multiple etiological factors and occur with signs and symptoms affecting the joint related muscular and neuromuscular components (21-23). In the treatment of TMD, in order to provide preventive procedures, it is necessary to investigate factors that may lead to this disease especially during different dentition periods.

Regulation of the postural structure of the body is possible if the changes in the biological subunit reorganize the postural control systems $(11,13)$. The neck muscles that are adjacent to the TMJ region play an important role in providing the balance of the head and stomatognathic muscles. It means that any level of differences in these structures can cause changes throughout the whole complex. The masticatory muscles can be affected by alterations in head posture and vice versa. Thus, any manipulation of the mandibular muscles can lead to changes in head posture $(13,24)$ and changes in the cervical spine structure can also play a role in developing TMD (13).

As the TMJ region is directly adjacent to the cervical and scapular regions, postural changes in the upper neck and head region correlate with TMD (13). Gonzalez and Manns (25) stated that FHP was caused by an extension of the upper cervical spine (C1-C3) and a flexion of the lower cervical spine (C4-C7) called hyperlordosis. The authors also emphasized that hyperextension was observed in the head and upper cervical spinal region in TMD patients. Alarcón et al. (26) suggested that the position of the jaw can affect the muscles in the peripheral region and cause postural adaptations at the spine level. Asymmetric malocclusions such as unilateral cross bite have been reported to be a risk factor for unbalanced muscle activity and postural dysfunction. Similarly, Solow and Sonnesen (27) stated that there is a strong relationship between the cervical lordosis grade and vertical craniofacial morphology, overjet, class 2 and class 3 anomalies. It is thought that TMD and postural irregularities are related and risk factors for each other. In this way, it can be stated that there is a positive relationship between head/body posture and TMD $(8,9,11)$. In the present study, the aim was to investigate the relationship between TMD and head/body posture and this relationship was examined in different dentition periods. The presence of disk displacement, joint sounds, muscular irregularities, movement limitations and pain were accepted as TMD. In postural status evaluation, FHP and DSL were examined.

In order to determine the postural status, several techniques (e.g. surface electromyography, kinesiography, different clinical and instrumental posturographic approaches) have been used over the years (28). Despite some studies on the stomatognathic system and its relationship with posture (29-33), they have restrictive factors to their clinical application because of the absence of normative values for age, sex, weight, height and facial

Table IV. The relationship between postural irregularities and TMD

\begin{tabular}{|c|c|c|c|c|c|c|c|c|c|}
\hline & & \multicolumn{6}{|c|}{ TMD } & \multirow{2}{*}{\multicolumn{2}{|c|}{ Statistical analysis }} \\
\hline & & \multicolumn{2}{|c|}{ Absence } & \multicolumn{2}{|c|}{ Presence } & \multicolumn{2}{|c|}{ Total } & & \\
\hline & & $\mathbf{n}$ & $\%$ & $\mathbf{n}$ & $\%$ & $\mathbf{n}$ & $\%$ & Chi-square & $p$ value \\
\hline \multirow{3}{*}{ FHP } & Absence & 1137 & 87.9 & 157 & 12.1 & 1294 & 100 & \multirow{3}{*}{31.051} & \multirow{3}{*}{$0.000^{*}$} \\
\hline & Presence & 53 & 65.4 & 28 & 34.6 & 81 & 100 & & \\
\hline & Total & 1190 & 86.5 & 185 & 13.5 & 1375 & 100 & & \\
\hline \multirow{3}{*}{ DSL } & Absence & 1136 & 87.8 & 158 & 12.2 & 1294 & 100 & \multirow{3}{*}{27.423} & \multirow{3}{*}{$0.000^{*}$} \\
\hline & Presence & 54 & 66.7 & 27 & 33.3 & 81 & 100 & & \\
\hline & Total & 1190 & 86.5 & 185 & 13.5 & 1375 & 100 & & \\
\hline
\end{tabular}

TMD: Temporomandibular disorders, FHP: Forward head posture, DSL: Different shoulder levels, *Statistically significant difference 
morphology. For these reasons, this study was carried out in an out-of-clinical setting and simple photographic techniques were used as an evaluation criterion.

In the present study, the presence of TMD was found to be statistically significant $(p<0.05)$ in those individuals with FHP and DSL in mixed dentition. In permanent dentition, an association with both postural parameters and TMD was not observed as statistically significant. Similar to the findings of this study, it has been reported that TMD is not frequently observed during primary dentition, while it is increasingly observed during mixed dentition (7). Chaves et al. (34) reported that the alterations in head position were observed in $56 \%$ of cases of moderate to severe TMD in individuals in the 10-18 years age group. Cortese et al. (35) stated that FHP was one of the most common postural anomalies at 10-15 years of age, and this was a risk factor for TMD. It seems that there is a need for more standardized studies concerning the relationship between postural irregularities and TMD in different dentition periods in children.

Patients in the mixed dentition period should be carefully assessed for the presence of TMD and postural irregularities, since TMJ related diseases are expected to increase especially after the primary dentition period. It has been reported that TMD stimulate the effects of masticatory muscles on the compensator mechanism and this biomechanical adaptation pulls the shoulders upwards (34). The occurrence of postural disorders is believed to be due to excessive stresses on the cervical muscles depending on the increased activity of the masticatory muscles to compensate for joint disease in TMD individuals (34). Nicolakis et al. (9) reported that training for postural correction in those patients with TMJ disk displacement have had promising results. Consequently, it is not certain that TMD is caused by postural changes or postural disorders are caused by TMD (36). In this respect, both situations should be considered as a potential risk factor for each other. These patients should be diagnosed and treated as early as possible by medical doctors, pediatricians and pediatric dentists using multidisciplinary approaches.

\section{Conclusion}

TMD is not only a problem affecting adults but can also be seen in pediatric patients. However, when considering the relationship between postural status and changes and presence of TMD, it is unclear which one is the etiological factor for the other. The disorders mentioned should be diagnosed at the youngest possible age. This approach will be beneficial in terms of the elimination of both TMD and postural irregularities. Therefore, it is recommended that pediatric dentists and pediatricians should evaluate the findings that may raise suspicions about the diagnosis of TMD and postural irregularities in a multidisciplinary manner.

\section{Ethics}

Ethics Committee Approval: The study protocol was approved by the Ethics Committee of Ankara University (approval number: 150/2).

Informed Consent: Informed consent was obtained by the parents.

Peer-review: External and internal peer-reviewed.

\section{Authorship Contributions}

Surgical and Medical Practices: C.D., N.Ö., Concept: C.D., A.D., N.Ö., Design: C.D., A.D., N.Ö., Data Collecting or Processing: C.D., N.Ö., Analysis or Interpretation: C.D., A.D., N.Ö., Literature Search: C.D., A.D., N.Ö., Writing: A.D., N.Ö.

Conflict of Interest: No conflict of interest was declared by the authors.

Financial Disclosure: The authors declared that this study received no financial support.

\section{References}

1. Alomar X, Medrano I, Cabratosa I, Clavero JA, Lorente M, Serra I, Monill JM, Salvador A. Anatomy of the temporomandibular joint. Semin Ultrasound CT MR 2007;28:170-83.

2. Gauer RL, Semidey MJ. Diagnosis and treatment of temporomandibular disorders. Am Fam Physician 2015;91:378-86.

3. Stohler CS. Muscle-related temporomandibular disorders. I Orofac Pain 1999;13:273-84.

4. Okeson JP. The classification of orofacial pains. Oral Maxillofac Surg Clin North Am 2008;20:133-44.

5. Greene CS. The etiology of temporomandibular disorders: implications for treatment. J Orofac Pain 2001;15:93-105.

6. Poveda Roda R, Bagan JV, Díaz Fernández JM, Hernández Bazán $\mathrm{S}$, Jiménez Soriano Y. Review of temporomandibular joint pathology. Part I: classification, epidemiology and risk factors. Med Oral Patol Oral Cir Bucal 2007;12:292-8.

7. Köhler AA, Helkimo AN, Magnusson T, Hugoson A. Prevalence of symptoms and signs indicative of temporomandibular disorders in children and adolescents. A cross-sectional epidemiological investigation covering two decades. Euro Arch Paed Dent 2009;10:16-25.

8. Lee WY, Okeson JP, Lindroth J. The relationship between forward head posture and temporomandibular disorders. I Orofac Pain 1995;9:161-7.

9. Nicolakis P, Nicolakis M, Piehslinger E, Ebenbichler G, Vachuda M, Kirtley C, Fialka-Moser V. Relationship between craniomandibular disorders and poor posture. Cranio 2000;18:106-12.

10. Strini PI, Machado NA, Gorreri MC, Ferreira Ade F, Sousa Gda C, Fernandes Neto Al. Postural evaluation of patients with temporomandibular disorders under use of occlusal splints. I Appl Oral Sci 2009;17:539-43.

11. Saito ET, Akashi PM, Sacco Ide C. Global body posture evaluation in patients with temporomandibular joint disorder. Clinics (Sao Paulo) 2009;64:35-9.

12. Motta L, Fernandes K, Ferrari R, Gonzalez D, Bussadori S. Temporomandibular dysfunction and cervical posture and occlusion in adolescents. Braz Oral Res 2012;11:401-5. 
13. Khan MT, Verma SK, Maheshwari S, Zahid SN, Chaudhary PK. Neuromuscular dentistry: Occlusal diseases and posture. I Oral Biol Craniofac Res 2013;3:146-50.

14. Cuccia A, Caradonna C. The relationship between the stomatognathic system and body posture. Clinics (Sao Paulo) 2009;64:61-6.

15. Nakahara H, Nakasato N, Kanno A, et al. Somatosensoryevoked fields for gingiva, lip, and tongue. I Dent Res 2004;83:307-11.

16. McNeely ML, Armijo Olivo S, Magee DJ. A systematic review of the effectiveness of physical therapy interventions for temporomandibular disorders. Phys Ther 2006;86:710-25.

17. Sari S, Sonmez H, Oray GO, Camdeviren H. Temporomandibular joint dysfunction and occlusion in the mixed and permanent dentition. J Clin Pediatr Dent 1999;24:59-62.

18. Sönmez H, Sari S, Oksak GO, Camdeviren H. Prevalence of temporomandibular dysfunction in Turkish children with mixed and permanent dentition. J Oral Rehabil 2001;28:280-5.

19. Farsi NM. Symptoms and signs of temporomandibular disorders and oral parafunctions among Saudi children. I Oral Rehabil 2003;30:1200-8.

20. Barbosa Tde S, Miyakoda LS, Pocztaruk Rde L, Rocha CP, Gavião MB. Temporomandibular disorders and bruxism in childhood and adolescence: review of the literature. Int I Pediatr Otorhinolaryngol 2008;72:299-314.

21. Conti PC, dos Santos CN, Kogawa EM, de Castro Ferreira Conti AC, de Araujo Cdos R. The treatment of painful temporomandibular joint clicking with oral splints: a randomized clinical tiral. J Am Dent Assoc 2006;137:1108-14.

22. Jerjes W, Upile T, Abbas S, et al. Muscle disorders and dentitionrelated aspects in temporomandibular disorders: controversies in the most commonly used treatment modalities. Int Arch Med 2008;1:23.

23. Gurbuz O, Kursoglu P, Alatas G, Altinbas K. The prevalence of temporomandibular disorder signs in people with mental retardation. J Oral Rehabil 2010;37:834-9.

24. Huggare JA, Raustia AM. Head posture and cervicovertebral and craniofacial morphology in patients with craniomandibular dysfunction. Cranio 1992;10:173-7.
25. Gonzalez HE, Manns A. Forward head posture: its structural and functional influence on the stomatognathic system, a conceptual study. Cranio 1996;14:71-80.

26. Alarcón JA, Martín C, Palma JC. Effect of unilateral posterior crossbite on the electromyographic activity of human masticatory muscles. Am / Orthod Dentofacial Orthop 2000;118:328-34.

27. Solow B, Sonnesen L. Head posture and malocclusions. Eur J Orthod 1998;20:685-93.

28. Manfredini D, Castroflorio T, Perinetti G, Guarda-Nardini L. Dental occlusion, body posture and temporomandibular disorders: where we are now and where we are heading for. I Oral Rehabil 2012;39:463-71.

29. Castroflorio T, Icardi K, Torsello F, Deregibus A, Debernardi C, Bracco P. Reproducibility of surface EMG in the human masseter and anterior temporalis muscle areas. Cranio 2005;23:130-7.

30. Castroflorio T, Farina $D$, Bottin A, Piancino MG, Bracco $P_{i}$ Merletti R. Surface EMG of jaw elevator muscles: effect of electrode location and inter-electrode distance. J Oral Rehabil 2005;32:411-7.

31. Castroflorio T, Bracco P, Farina D. Surface electromyography in the assessment of jaw elevator muscles. I Oral Rehabil 2008;35:638-45.

32. Leitner C, Mair P, Paul B, Wick F, Mittermaier C, Sycha T, Ebenbichler $G$. Reliability of posturographic measurements in the assessment of impaired sensorimotor function in chronic low back pain. J Electromyogr Kinesiol 2009;19:380-90.

33. Suvinen $\mathrm{TI}$, Malmberg J, Forster C, Kemppainen P. Postural and dynamic masseter and anterior temporalis muscle EMC repeatability in serial assessments. J Oral Rehabil 2009;36:814-20.

34. Chaves PJ, de Oliveira FEM, Damázio LCM. Incidence of postural changes and temporomandibular disorders in students. Acta Ortop Bras 2017;25:162-4.

35. Cortese S, Mondello A, Galarza R, Biondi A. Postural alterations as a risk factor for temporomandibular disorders. Acta Odontol Latinoam 2017;30:57-61.

36. Olivo SA, Bravo J, Magee DJ, Thie NM, Major PW, Flores-Mir $C$. The association between head and cervical posture and temporomandibular disorders: a systematic review. I Orofac Pain 2006;20:9-23. 